\title{
Autoionization widths of cold Rydberg atomic complexes
}

\author{
M.S. Dimitrijević ${ }^{1,2}$, V.A. Srećković ${ }^{3}$, Alaa Abo Zalam ${ }^{4,5}$, \\ K. Miculis ${ }^{6,7}$, D.K. Efimov ${ }^{8}$, N.N. Bezuglov ${ }^{4}$ and A.N. Klyucharev ${ }^{4}$ \\ ${ }^{1}$ Astronomical Observatory, Volgina 7, 11060 Belgrade, Serbia, (E-mail: \\ mdimitrijevic@aob.rs) \\ 2 Sorbonne Université, Observatoire de Paris, Université PSL, CNRS, \\ LERMA, F-92190, Meudon, France \\ 3 Institute of Physics Belgrade, Pregrevica 118, 11080 Zemun, Belgrade, \\ Serbia \\ 4 Saint Petersburg State University, 7/9 Universitetskaya nab., St. Petersburg, \\ 199034, Russia \\ 5 Physics Department, Faculty of Science, Menoufia University, Menoufia, \\ Egypt \\ ${ }^{6}$ University of Latvia, Institute of Atomic Physics and Spectroscopy, LV-1586 \\ Riga, Latvia \\ 7 Moscow State Engineering Physics Institute, Kashirskoe shosse 31, Moscow \\ 115409, Russia \\ 8 Instytut Fizyki im. Mariana Smoluchowskiego, Uniwersytet Jagielloski, \\ 30-348 Krakw, Poland
}

Received: July 10. 2019; Accepted: August 22, 2019

\begin{abstract}
We have considered two types of interactions between particles resulting in autoionization of the intermediate Rydberg collisional complex. The first type is caused by the charge exchange processes inside the ionic core of the complex and is responsible for the formation of the final associative ionization channel due to the departure of a Rydberg electron (RE) of the complex to the energy continuum. We have investigated the evolution of the orbital momentum $L$ of $\mathrm{RE}$ and discovered a non-trivial time dependence of $L$, demonstrating a contrasting oscillatory structure in both direction orientation and its absolute value. The second type of interaction, dipole-dipole interaction, dominates in cold highly excited gaseous media and leads to Penning ionization of various pairs of Rydberg atoms. We have found the optimal quantum configurations of atomic pairs, which intensify the PI rates by several orders of magnitude, and become important suppliers of charged particles upon the evolution of Rydberg gas into cold plasma.
\end{abstract}

Key words: Atomic processes - Line: profiles - Plasmas 


\section{Introduction}

In scientific literature, up until the early 1980s, astrophysical research largely focused on such global issues as star formation problems, planetary nebulae, and interstellar gas physics (Dalgarno, 1975). Currently, the subject of elementary collisional processes involving light and matter particles in the geospace environment is attracting more and more attention (see the results of the space mission 'Galileo Mission to Jupiter 1989-2003', Lopes \& Williams 2005). Modern experimental techniques make it possible to compare the rate constants of various reactions obtained in the geospace environment with their terrestrial analogues (Gnedin et al., 2009), for example, for ion-recombination collisions in cold helium-saturated atmospheres of cooling stars of the White Dwarf type (DB White dwarfs, Mihajlov et al., 2003). Recombination processes result in populating highly excited states and are the important sources of the formation of Rydberg atoms/molecules in stellar atmospheres of late spectral types, interstellar nebulae and other space objects, including our solar system (see, for instance, Dalgarno 1975; Graham et al. 2012). The appearance of Rydberg particles plays a fundamental role in the Earth's lower ionosphere. In many situations (e.g., when solar flares occur) it exerts an uncontrollable influence on the propagation of satellite radio signals from the global positioning system (GPS, Buenker et al., 2013).

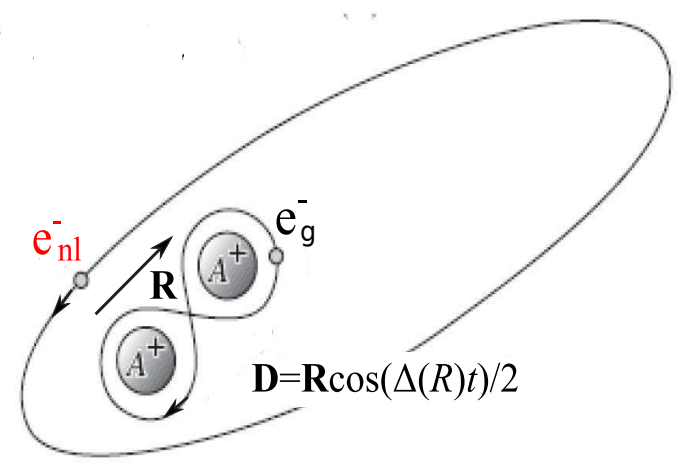

Figure 1. Schematic representation of the intermediate collisional complex $A_{2}^{* *}$ structure formed in the course of the reaction (1).

Autoionization processes of Rydberg atomic complexes $\mathrm{A}_{2}^{* *}$ are inverse to recombination channels and may occur as intermediate states

$$
\mathrm{A}^{* *}(n l)+\mathrm{A} \rightarrow \mathrm{A}_{2}^{* *} \rightarrow\left\{\begin{array}{l}
\mathrm{A}^{* *}\left(n^{\prime} l^{\prime}\right)+\mathrm{A}^{+}+e^{-} \\
\mathrm{A}_{2}^{+}+e^{-}
\end{array}\right.
$$


in various collisional events involving atom $\mathrm{A}$ in the normal state and Rydberg atom $\mathrm{A}^{* *}(n l)$ with the principal and orbital quantum numbers $n$ and $l$ respectively. The theory for describing processes (1), which was called dipole resonance ionization (DRI), was based on Fermi's proposal (Fermi, 1934) to consider the intermediate formation $\mathrm{A}_{2}^{* *}$ as a structure (see Fig. 1), consisting of positively charged atomic cores $\mathrm{A}^{+}$, generalized valence electron $e_{g}^{-}$of atom A and quasi-free Rydberg electron (RE) $e_{n l}^{-}$in a Keplerian orbit with binding en$\operatorname{ergy} \varepsilon_{n l}=1 / 2 n^{* 2}$, where $n^{*}$ is the effective quantum number. Within the DRI model, the probability of realization of various output channels (the Penning channel and the associative channel) of reaction (1) is determined by the internal dipole moment $\boldsymbol{D}$ of quasimolecule $\mathrm{A}_{2}^{* *}$. Moment $\boldsymbol{D}=\boldsymbol{R}(t) \cos (\Delta(R) t) / 2$ arises in the process of recharging in the system $\left(\mathrm{A}+\mathrm{A}^{+}\right)$when changes in both the internuclear distance $\boldsymbol{R}(t)$ and the recharge frequency $\omega(t)=\Delta(R(t))$ are adiabatically slow (Janev \& Mihajlov, 1980). Alternating dipole $\boldsymbol{D}$ induces a quasi-monochromatic field $\boldsymbol{E}(t)$ that perturbs RE motion $e_{n l}^{-}$and leads to its "photoionization" as soon as frequency $\Delta(R)$ becomes greater than the RE binding energy $\varepsilon_{n l}$. The system is traditionally described in the adiabatic approximation using the corresponding potential curves of Rydberg complex $\mathrm{A}_{2}^{* *}$ formed by $\mathrm{RE} e_{n l}^{-}$and molecular ion $\mathrm{A}_{2}^{+}$(see Fig. 2).

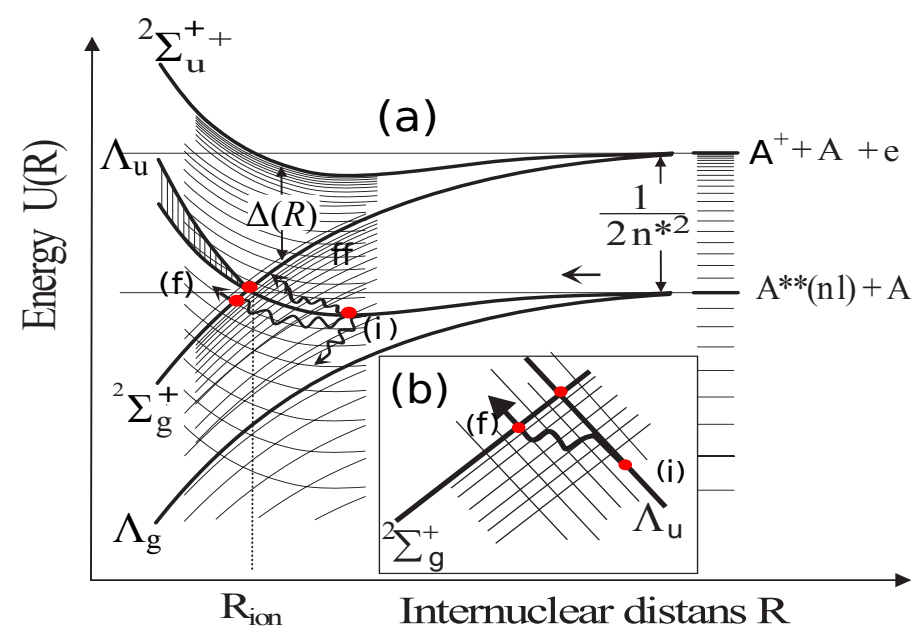

Figure 2. Illustration of the development of diffusion ionization in a quasi-molecule formed in the collision of two alkali metal atoms in the ground state A and in the highly excited state $\mathrm{A}^{* *}(n l)$. Box (b) shows one of the many possible evolutions of the initial excitation of a diatomic system.

Highly excited systems have specific features, when they interact with electromagnetic fields, due to the Coulomb condensation of quantum states near the 
energy continuum. Both the Stark and the Zeeman effects cause multiple quasiintersections of the Rydberg electron sublevels, which leads to RE chaotic motion along the dense grid of Rydberg states (see Fig. 2(b)) with the subsequent exit into the energy continuum. Such uncontrolled dynamic chaos (Zaslavsky, 2007) in the evolution of Hamiltonian systems is observed for single Rydberg atoms under the influence of external microwave fields (Koch \& Van Leeuwen, 1995; Krainov, 2010), as well as for collisional Rydberg complexes with a strong intra-atomic dipole interaction (Klyucharev et al., 2007). In the case of molecular systems, diffusion migration of initial excitation can lead to dissociation of molecules (Dashevskaya et al., 2002).

Various aspects of the autoionization processes of Rydberg atomic formations were considered in works Gnedin et al. (2009); Klyucharev et al. (2007). They demonstrate, in particular, the important role of the dynamic chaos development for various ionization phenomena occurring in cold astrophysical environments. In continuation of these studies, in this article we present new numerical and analytical data related to the following problems. (i) Analysis of the nontrivial temporal dynamics of the angular momentum $L(t)$ of a Rydberg electron in electromagnetic fields, and (ii) the influence of this dynamics on the efficiency of the diffusion ionization. (iii) Analysis of constant rates $\Gamma_{N}$ of the Penning autoionization of quasi-molecules formed by two cold Rydberg sodium atoms in different quantum states $N$, subject to the long-range dipole-dipole interaction.

Henceforth the atomic system of units is used, unless otherwise stated.

\section{The problem of the Rydberg electron orbital momentum conservation upon diffusion ionization}

The temporal evolution of the orbital momentum $\mathbf{L}$ of the Rydberg intermediate complex largely determines the character and properties of atom-atom collisions of type (1). In the quasi-classical approximation, the absolute value $\mathbf{L}$ is given by the relation $L=|\mathbf{L}|=l+0.5$, where the term 0.5 is called the Langer correction (Landau \& Lifshitz, 1977). In both the early Koch \& Van Leeuwen (1995); Delone et al. (1983) and comparatively recent papers Krainov (2010); Bezuglov et al. (2002), which laid foundation for the studies on the dynamic stochastization of highly excited states under the influence of microwave radiation, the authors relied on the important assumption of adiabatic invariance of $L$. This assumption was later questioned in Golubkov \& Devdariani (2011). Within the framework of the theory of a multichannel quantum defect, this work demonstrated a change in the orbital quantum number $l$ during inelastic collisions of heavy particles going through an intermediate state of a molecular ion. Another example refers to L-mixing processes in D-stratum of the Earth's atmosphere, which, inter alia, leads to occurrence of 'spectral windows' for incoherent radiation (Buenker et al., 2013). 
Noteworthy is an important result in Bezuglov et al. (2002), which allows us to reduce the analysis of the effects of $\mathrm{RE} e_{n l}^{-}$motion perturbation due to the intramolecular electric field $\mathbf{E}(t)$ (see the discussion of Fig. 1) to the case of a spatially homogeneous quasi-monochromatic microwave field influencing RE. This section investigates various aspects of the momentum $L$ conservation for the corresponding model Hamiltonian

$$
\begin{aligned}
H(\mathbf{p}, \mathbf{r}, t) & =\frac{\mathbf{p}^{2}}{2}-1 / r+(\mathbf{E}(t) \cdot \mathbf{r}) ; \\
\mathbf{E}(t) & =\mathbf{E}_{0} \cos \left(\omega_{0} t+\theta\right),
\end{aligned}
$$

which describes the interaction of a hydrogen atom with an external microwave field of frequency $\omega_{0}$ and vector amplitude $\mathbf{E}_{\mathbf{0}}$.

\subsection{The hydrogen atom in a slowly varying electric field}

The main features of the dynamics of the angular momentum $\mathbf{L}$ can be observed in a relatively simple and analytically solvable example of an almost stationary field ( $\omega_{0}$ is enough small - see below) with RE moving regularly. In the range of moderate amplitudes $E_{0}=\left|\mathbf{E}_{0}\right|$ (see below), it is convenient to find the evolution of vector $\mathbf{L}(t)$ in the adiabatic approximation by averaging the equation for the rate of change $d \mathbf{L} / d t$ along the Coulomb electron trajectory: $d / d t\langle\mathbf{L}\rangle=-\left[\langle\mathbf{r}\rangle \mathbf{E}_{0}\right]$ (symbol $\langle\ldots\rangle$ means averaging). The moderate disturbance implies minor changes in the parameters of the RE Keplerian orbits $\mathbf{r}(t)$ for the period $T=2 \pi n^{3}$ of classical motion. Here, a quasi-classical approach Landau \& Lifshitz (1977) is used, in which, in accordance with Bohr's correspondence principle, the state with quantum numbers $\{n, l, m\}$ is juxtaposed with the classical trajectory with the following motion integrals (Landau \& Lifshitz, 1976): energy $\varepsilon=-1 / 2 n^{2}$, the modulus of angular momentum $L=l+0.5$, and the projection $L_{p l}=m$ ( $m$ is azimuthal quantum number) on the direction of the polarization vector $\mathbf{e}_{p l}=\mathbf{E}_{0} / E_{0}$ of the external field.

It is easy to show that the mean value $\langle\mathbf{r}\rangle$ is directed along the semimajor axis (length $a=n^{2}$ ) of the Keplerian ellipse and, therefore, is expressed $\langle\mathbf{r}\rangle=$ $3 a \cdot\langle\mathbf{A}\rangle / 2$ in terms of the Runge-Lenz vector (Laplace integral) $\mathbf{A}=\left[\mathbf{v}_{e} \mathbf{L}\right]-$ $(\mathbf{r} / r)$ (Landau \& Lifshitz, 1976) where $\mathbf{v}_{e}$ is the RE velocity. The equation for evolution $\langle\mathbf{L}\rangle$, therefore, includes the average $\langle\mathbf{A}\rangle$, i.e.

$$
\frac{d\langle\mathbf{L}\rangle}{d t}=-\frac{3}{2} a\left[\boldsymbol{E}_{0}\langle\mathbf{A}\rangle\right] .
$$

Evolution $\langle\mathbf{A}\rangle$, in turn, is related to vector $\langle\mathbf{L}\rangle$ (Landau \& Lifshitz, 1976)

$$
\frac{d\langle\mathbf{A}\rangle}{d t}=-\frac{3}{2}\left[\boldsymbol{E}_{0}\langle\mathbf{L}\rangle\right] .
$$


Note that $d\langle\varepsilon\rangle / d t=0$, and the length $a=n^{2}=n_{0}^{2}$ of the semimajor axis undergoes no evolution and remains adiabatically unchanged. Hereinafter, index '0' indicates the original (unperturbed) values of physical quantities at the moment of switching an external field. The closed system of equations (4) and (5) is reduced to two independent equations after the transition to new variables $\mathbf{G}_{ \pm}$and the rescaling of time:

$$
\begin{gathered}
\mathbf{G}_{ \pm}=n_{0}\langle\mathbf{L}\rangle \pm n_{0}^{2}\langle\mathbf{A}\rangle ; \quad \tau=n_{0} t \\
\frac{d}{d \tau} \mathbf{G}_{ \pm}= \pm \frac{3}{2}\left[-\boldsymbol{E}_{0} \mathbf{G}_{ \pm}\right] .
\end{gathered}
$$

Equations (7) have an obvious geometric interpretation: vectors $\mathbf{G}_{ \pm}$rotate around vector $-\mathbf{E}_{0}$ in the opposite directions with the circular frequency of the same modulus $\Omega_{\tau}=(3 / 2) E_{0}$ in units $1 / \tau$ or $\Omega_{t}=(3 / 2) n_{0} E_{0}$ in units $1 / t$. The corresponding revolution time is $T_{E}=8 \pi /\left(3 n_{0} E_{0}\right)$. The validity range of equations (4)-(7) of our adiabatic model follows from two requirements: (i) smallness of rotation angles $\Delta \theta=\Omega_{t} T<2 \pi$ of vectors $\mathbf{G}_{ \pm}$for the RE period of motion $T=2 \pi n_{0}^{3}$ and (ii) smallness of the field frequency $\omega_{0}$ that may be expressed as

$$
\omega_{0}<1.5 E_{0} n_{0}<1 / n^{3} .
$$

Fields satisfying inequality (8) can be considered moderate, although they overlap the critical field value $E_{\mathrm{cr}}=n_{0}^{-4} / 16$, at which classical atom ionization occurs (Delone et al., 1983).

The modulus value $|\mathbf{A}|$ is equal to the eccentricity $|\mathbf{A}|=\sqrt{1-\left(L / n_{0}\right)^{2}}$ of the Keplerian orbit (Landau \& Lifshitz, 1976), and therefore for Rydberg states $\left(n_{0}>>1\right)$, the initial value $\left|\mathbf{A}_{0}\right|$ is close to unity. If we limit ourselves to the typical for experiments case of small values $l_{0} \sim 1<<n_{0}$, then

$$
\mathbf{G}_{+}^{0} \approx-\mathbf{G}_{-}^{0} \approx n_{0}^{2} \mathbf{A}_{0}
$$

as follows from (6). It can be seen that, due to the initial configuration of $\mathbf{G}_{ \pm}^{0}$ (9), the dynamics (7) of vectors $\mathbf{G}_{ \pm}$is determined by the mutual orientation of vectors $\mathbf{A}_{0}$ and $\mathbf{E}_{0}$.

At the initial time $(t=0)$, vectors $\mathbf{G}_{ \pm}$according to (9) are virtually directed in opposite directions, which gives $\left|\mathbf{L}_{0}\right|=\left|\mathbf{G}_{+}^{0}+\mathbf{G}_{-}^{0}\right| / 2 n_{0}=l_{0}+0.5 \approx 1$. With an increase in time $t$ due to rotation around $\mathbf{E}_{0}$, vectors $\mathbf{G}_{ \pm}$approach each other. After a quarter of a turn at time $t=T_{E} / 4$ the modulus of their halfsum, i.e. $n_{0}|\mathbf{L}|$, reaches the maximum value $\max \left(n_{0}|\mathbf{L}|\right) \approx n_{0}^{2} \sin \varphi$, where $\varphi$ is the angle between the directions of vectors $\mathbf{A}_{0}$ and $-\mathbf{E}_{0}$. Then the orbital momentum $\mathbf{L}$ will be almost orthogonal to the plane with vectors $\mathbf{A}_{0}$ and $\mathbf{E}_{0}$. If $\varphi=\pi / 2$, the RE orbit becomes quasi-circular, because eccentricity $|\mathbf{A}|=$ $\sqrt{1-\left(L / n_{0}\right)^{2}}=0$. With time $t$, vectors $\mathbf{G}_{ \pm}$begin to diverge. When $t=T_{E} / 2$ they acquire the opposite orientation again in the plane that is orthogonal to vector $\mathbf{E}_{0}$; the corresponding projections of $\mathbf{G}_{ \pm}$change their signs as compared 
to the initial values when $t=0$. As a result, the angular momentum $\langle\mathbf{L}\rangle$ takes the position of vector $\mathbf{L}_{0}$ rotated around $\mathbf{E}_{0}$ by 180 degrees. Therefore $|\mathbf{L}|(t=$ $\left.T_{E} / 2\right)=L_{0}$. The same directions of $\mathbf{G}_{ \pm}$correspond to moment $t=3 / 4 T_{E}$, and $|\mathbf{L}| \approx n_{0} \sin \varphi$. Finally, at the end of the rotation period $t=T_{E}$, vectors $\mathbf{A}_{0}, \mathbf{L}_{0}$ occupy their initial ' 0 ' positions.

Fig. 3 illustrates the conclusions made about the character of the motion of $\mathrm{RE}$ in the initial state of $10 \mathrm{p}\left(n_{0}=10, l_{0}=1\right)$. Vectors $\mathbf{A}_{0}, \mathbf{E}_{0}, \mathbf{L}_{0}$ are chosen in a mutually orthogonal way, so that the RE orbit remains in (X, Y)plane containing vectors $\mathbf{A}_{0}, \mathbf{E}_{0}$ with the angle between them $\varphi=\pi / 2$. The direction of the momentum $\mathbf{L}$ remains unchanged and coincides with $\mathrm{Z}$-axis. In Fig. 3(a) the periodic oscillations of $\mathbf{L}_{Z}$ projection within the segment $\left(-n_{0}-\right.$ $\left.0.5, n_{0}+0.5\right)=(-10.5,10.5)$ can be clearly seen. The corresponding conditionally periodic motion of RE (Fig. 3(b)) changes from quasi-circular $\left(\mathbf{L}_{Z}= \pm 10.5\right)$ to quasi-rectilinear $\left(\mathbf{L}_{Z}=0\right)$ with a RE passing through the Coulomb center and changing the direction of rotation.
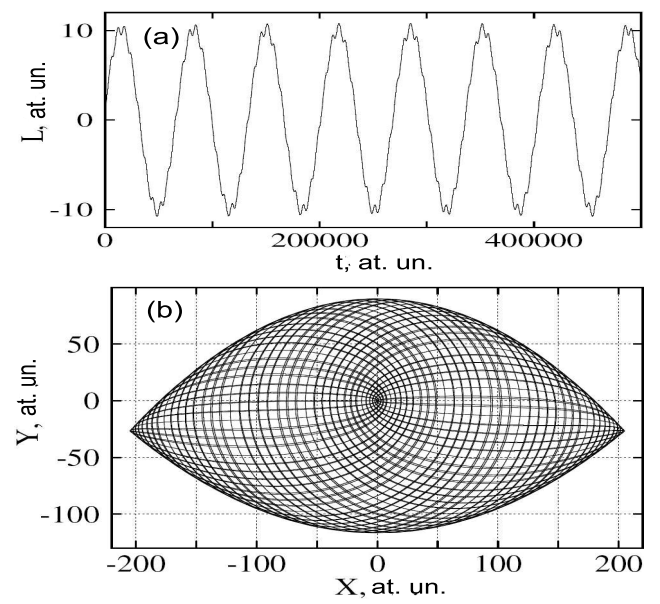

Figure 3. Time behavior of orbital momentum $\mathrm{L}=\mathbf{L}_{Z}$ (a) and electron path (b) in a steady electric field $\left(\omega_{0}=0\right)$ with $\mathrm{E}_{0}=0.98 /\left(16 n_{0}^{4}\right)$ amplitude and $\mathbf{A}_{0} \perp \mathbf{E}_{0}$ configuration. RE initial state is $10 \mathrm{p}$.

Another simple case of a two-dimensional motion of a RE in (X, Y)-plane is the situation of $\varphi=0$, i.e. when $\mathbf{A}_{0} \| \mathbf{E}_{0}$ and momentum $\mathbf{L}$ is directed along the $\mathrm{Z}$-axis. If the $\mathrm{X}$-axis is directed along vector $\mathbf{A}_{0}$, then it immediately follows from equation (5) that the increment of the current value of vector $\langle\mathbf{A}\rangle$ occurs along the Y-axis. As a result, the solution of the system of equations (6) and (7) is given by the following simple harmonic oscillations of vectors $\langle\mathbf{L}\rangle,\langle\mathbf{A}\rangle$

$$
\langle\mathbf{L}\rangle=\mathbf{L}_{0} \cos \left(1.5 n_{0} E_{0} t\right)
$$




$$
\langle\mathbf{A}\rangle=\mathbf{A}_{0}-\left(L_{0} / n_{0}\right) \sin \left(1.5 n_{0} E_{0} t\right) \mathbf{e}_{Y} .
$$

Evolution (10)-(11) corresponds to the periodic 'pinching' of Rydberg trajectories $\left(n_{0}>>1\right)$ : the semi-major axis slightly changes its direction, while vector $\mathbf{L}$ turning to zero transforms the orbital into a quasi-straight line.

Noteworthy, the appearance of an additional rotational motion of RE around the direction of the external electric field with frequency $\Omega_{t}$ causes, according to Bohr's correspondence principles, the equidistant splitting of energy levels with the step size $\Delta \varepsilon=\Omega_{t}=(3 / 2) n_{0} E_{0}$. This result is known from the quantum theory of the linear Stark effect (Landau \& Lifshitz, 1977).

\subsection{The case of stochastic motion in a microwave field}

In the study of evolution of Rydberg states of a hydrogen atom interacting with a microwave field (Koch \& Van Leeuwen, 1995; Krainov, 2010; Delone et al., 1983), approximation of conservation of momentum $\mathbf{L}$ considerably facilitates the task due to the reduction of tridimensional RE dynamics to the issue of RE stochastic migration along a one-dimensional atomic level energy grid (Krainov, 2010). In the previous section, it was shown that the external field leads to a complex evolution of the RE Keplerian orbits, transferring them from the initially strongly elongated orbits to the quasi-circular ones and vice versa. Even with relatively small principal quantum numbers $\left(n_{0} \sim 10\right)$, the angular momentum modulus $\langle\mathbf{L}\rangle$ can vary significantly up to values $n_{0} \sin \varphi$.

This section will demonstrate the notable changes of $\mathbf{L}$ in microwave fields in the context of dynamic chaos conditions development and examine the validity of a one-dimensional model of diffusion ionization application. We shall start with an overview of key ideas of publications Krainov (2010); Delone et al. (1983) drawing upon the adiabatic constancy of RE orbital momentum $|\mathbf{L}|$.

(i) Global chaos is a threshold process for field amplitude $E_{0}$ and occurs when

$$
E_{0}>E_{c} \approx \frac{1}{n_{0}^{4}} \frac{1}{\chi_{l}\left(n_{0}^{3} \omega_{0}\right)^{1 / 3}} ; \quad L \leq L_{c} \approx\left(3 / \omega_{0}\right)^{1 / 3} .
$$

Values of $\chi_{l}$ parameter in $L \leq L_{c}$ region vary gradually from $\chi_{0} \approx 35$ to $\chi_{l_{c}} \approx 22$. Value (12) for $E_{c}$ critical amplitude was obtained for parameter $s=\omega_{0} n_{0}^{3}>3$, i.e. under the condition of substantial non-adiabaticity of the excitation by the microwave field (see Eq. (8)).

(ii) When $E_{0}$ is a fixed value, expression $(12)$ sets a $n_{c} \approx \sqrt[4]{1 /\left(\chi_{l} E_{0}\right)}$ limit for the values of principal quantum numbers $n$ separating the areas of RE random $\left(n>n_{c}\right)$ and regular $\left(n<n_{c}\right)$ motion. For the initial condition $n_{0}>n_{c}$, publications Dashevskaya et al. (2002); Bezuglov et al. (2001) estimated the related time $\tau_{D I}$ of diffusion ionization as

$$
\tau_{D I}\left(n_{0}\right) \approx 2 \omega_{0}^{4 / 3} /\left(n_{0} E_{0}^{2}\right),
$$

which, similarly to relation (12), does not depend on $L$ when $L \leq L_{c}$. 
(iii) When the current value of orbital momentum $L(t)>L_{c} \sim(3 / s)^{1 / 3} n_{0} \sim$ $n_{0}$ (which corresponds to RE orbits approximating to a circular orbit), dynamic resonance configuration peculiarities arise (Krainov, 2010; Delone et al., 1983). Critical field values $E_{c}$ in $L>L_{c}$ area increase drastically with practical blocking of the dynamic chaos condition and, accordingly, prohibition of diffusion ionization.

Analysis of phenomena with strong path stochastization requires the use of reliable tools for numerical computation of particle parameters. Such tools include symplectic integrators of motion equations (Hairer, 1999) for various physical problems, including complex integro-differential tasks related to the radiation energy transfer processes in optically dense media (Kazansky et al., 2001) with a multi-level structure of atomic quantum states (Sydoryk et al., 2008; Kirova et al., 2017). A numeric algorithm of advanced stability for analyzing stochastic migration effects was suggested in publication Efimov et al. (2014). This algorithm is based on a combination of a symplectic integrator variety, also known as the split propagation technique, and Floquet technique (Chu \& Telnov, 2004).
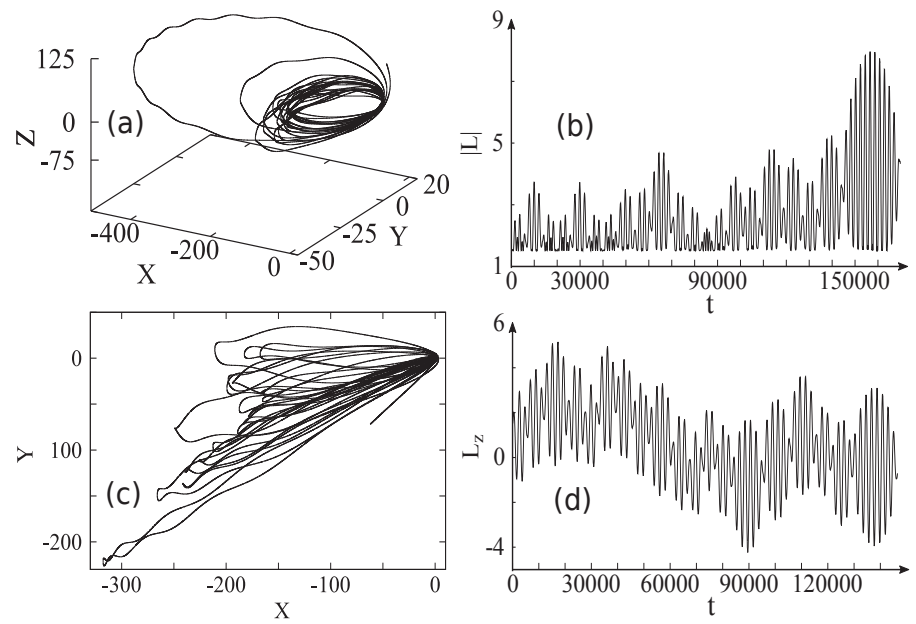

Figure 4. Rydberg electron trajectories in a hydrogen atom (boxes (a), (c)) and the related time behavior of the orbital momentum (boxes (c), (d)) for 10p initial state, at a microwave field frequency of $\omega_{0}=3 / 10^{3}$. Axial values are quoted in at. un.

The data obtained in Efimov et al. (2014) reflect invalidity of the approximation concerning invariance of angular momentum L. As an example, motion paths and temporal evolution of orbital momentum under the conditions of diffusion ionization manifestation are shown in Fig. 4. We used the electron initial 
state in a hydrogen atom equal to $10 \mathrm{p}\left(n_{0}=10, l_{0}=1\right)$. An electron is exposed to the impact of a $\omega_{0}=3 / 10^{3}$ frequency microwave field whose amplitude $E_{0}$ by far exceeds the threshold value $E_{c}(12)$. Two typical initial configurations of $\mathbf{E}_{0}$ and $\mathbf{A}_{0}$ vectors were selected intentionally at $\varphi=\pi / 2$ angle, which corresponds to the maximum change (see previous section 2.1) in $|\mathbf{L}|$ module or its projection $\mathrm{L}_{Z}$ for tridimensional $\left(E_{0}=6.5 E_{c}\right.$, Fig. 4, boxes (a), (b)) and bidimensional $\left(E_{0}=8.2 E_{c}\right.$, Fig. 4 , boxes $\left.(\mathrm{c}),(\mathrm{d})\right)$ paths respectively. All time dependencies are traceable until RE escape into the energy continuum. The results of the numerical calculations shown demonstrate a noticeable decrease in $\mathbf{L}$ oscillation amplitude in the microwave field when global chaos occurs compared to regular motion (cf. boxes (a) in Fig. 3 and (d) in Fig. 4 for equal type configurations of the electric field and the electron initial state).
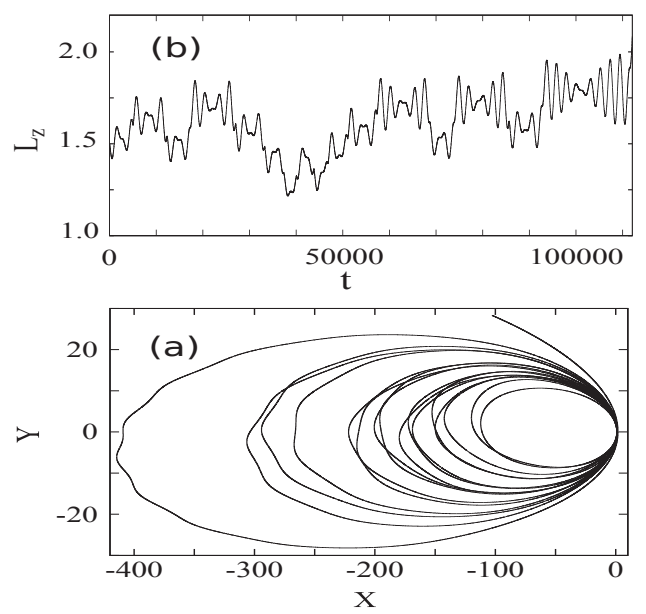

Figure 5. Similar to Fig. 3 for $\mathbf{A}_{0} \| \mathbf{E}_{0}$ configuration and microwave field choice at frequency $\omega_{0}=3 / 10^{3}$ and amplitude $E_{0}=4.7 E_{c}$.

RE random drift blocking starts when $L>L_{c} \approx\left(3 / \omega_{0}\right)^{1 / 3}$. For the examples shown in Fig. 4 this corresponds to $L_{c} \approx 10$ value, i.e. $L$ never exceeds the value $\approx 2 / 3 L_{c}$ (see Fig. 4(b),(d)). Under the conditions of stochastic process development with $L<L_{c}$, the dependence of all diffusion factors on $L$ appears to be weak (Krainov, 2010). To this extent the key conclusions from the monodimensional model of diffusion ionization suggested in publications Krainov (2010); Delone et al. (1983) retain their significance, since within the $\tau_{D I}$ time lapse (13) $L$ values remain within $L_{c}$ limits.

It should be noted that an exceptional configuration for $\mathbf{A}_{0} \| \mathbf{E}_{0}$ vectors $(\varphi=0)$ in $(\mathrm{X}, \mathrm{Y})$ plane and $\mathbf{L}=\mathrm{L}_{Z} \mathbf{e}_{Z}$ momentum exists. As follows from Fig. $5, \mathrm{~L}_{Z} \approx$ const $(=1.5$ ) approximation (box (a)) quite successfully matches 
RE bidimensional motion (box (b)). The same configuration in the case of an adiabatic field $\left(\omega_{0} \approx 0\right)$ and a regular motion demonstrates significantly larger oscillations (10) of $\mathrm{L}_{Z}$ that lies within the interval $(-1.5,1.5)$.

\section{Autoionization widths of quasi-molecules formed by pairs of cold Rydberg atoms}

Cold media are currently seen as promising physical objects in connection with the solution of a number of interdisciplinary applied problems, including both the issues of quantum information processing (Saffman et al., 2010) and the physics of the unique ultra-cold plasma creation (Lyon \& Rolston, 2016). Ultracold plasma is formed in magneto-optical traps in the 'frozen' Rydberg gas of alkali-metal atoms excited to the initial states with the principal quantum number of $n_{0} \sim 50$, concentration $\rho_{0}>5 \cdot 10^{10} \mathrm{~cm}^{-3}$ and several $\mu \mathrm{K}$ temperature. It should be noted that ultra-cold plasma formation occurs through a diffusion stage (Tanner et al., 2008) (see Fig. 6), which features formation of highly asymmetric Rydberg atomic pairs with different orbital $l$ and principal quantum $n$ numbers due to collisional processes.

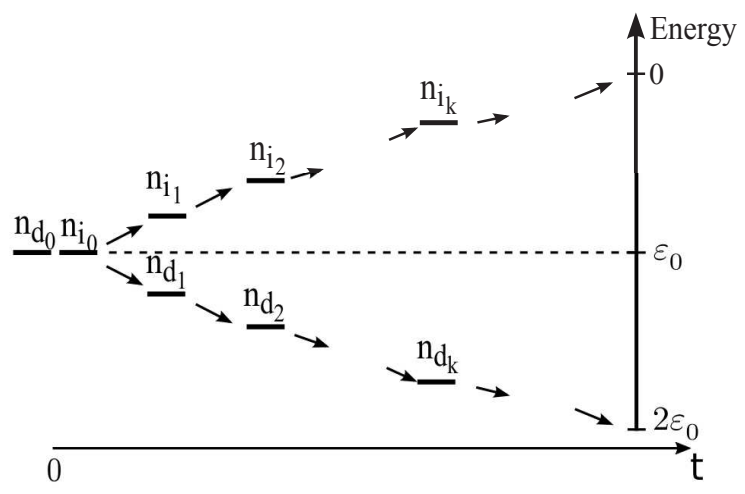

Figure 6. (Adopted from Tanner et al. 2008) Schematic of population diffusion over a band of energies for atomic pairs. The energy $\varepsilon_{i d}=\varepsilon_{n_{d}}+\varepsilon_{n_{i}}$ of the $n_{d} n_{i}$ pairs of atoms initially identically excited $\left(n_{d_{0}}=n_{i_{0}}=n_{0}\right)$ maintains its initial value $2 \varepsilon_{0}=-1 / 2 n_{0}^{* 2}$ because of the near resonance coupling between the two-atom quasimolecules $\tilde{n}_{d} \tilde{n}_{i}$. The energy axis corresponds to the individual atomic energies $\varepsilon_{n_{d}}, \varepsilon_{n_{i}}$.

The Penning ionization should be considered to be the most important type of collisional processes that create primary charged particles in cold media (Fig. 7).

$$
\mathrm{A}^{* *}\left(n_{d} l_{d}\right)+\mathrm{A}^{* *}\left(n_{i} l_{i}\right)=\mathrm{A}^{* *}\left(n_{d}^{\prime} l_{d}^{\prime}\right)+\mathrm{A}^{+}+e\left(\varepsilon_{i}^{\prime} l_{i}^{\prime}\right) .
$$




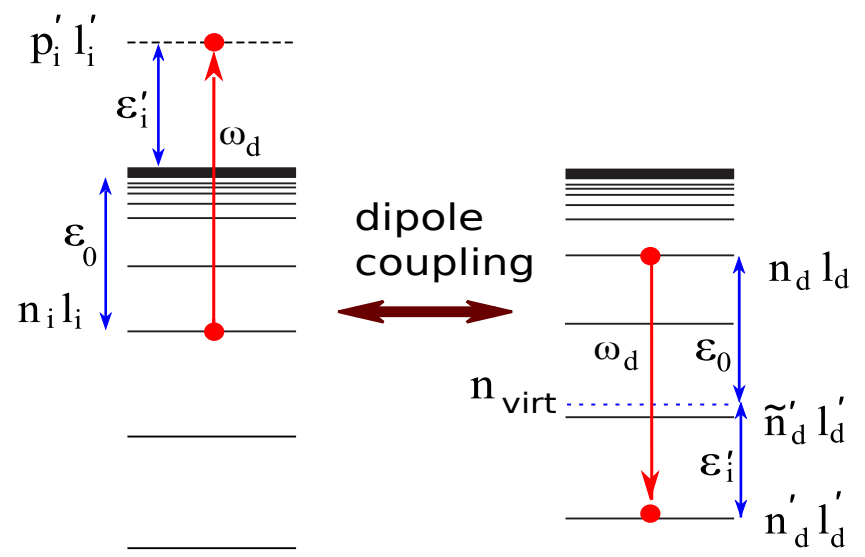

Figure 7. Auger-process illustration for resonance interaction between a Rydberg atomic pair $\left\{\mathrm{Ai}^{* *}, \mathrm{Ad}^{* *}\right\}$ and the energy continuum. The partial transition $N \rightarrow$ $N^{\prime}$ is presented.

Contrary to the associative ionization, the Penning ionization (PI) is a nothreshold reaction. Long-range dipole-dipole interaction between two Rydberg alkali-metal atoms results in a free electron escaping due to the Auger process: one of the atoms (d) undergoes a transition from state $n_{d} l_{d}$ to a deeper bound state $n_{d}^{\prime} l_{d}^{\prime}$, while the other atom (i) gains the released energy and jumps from state $n_{i} l_{i}$ to the continuum state $\varepsilon_{i}^{\prime} l_{i}^{\prime}$.

In this chapter, we analyze some nontrivial features of PI in Rydberg gases, which make PI an important source of charged particles in cold media.

\subsection{Penning ionization (PI) cross sections}

Our method for calculating PI cross section $\sigma_{P I}$ for reaction (14) is based on the Katsuura-Smirnov model (Katsuura, 1965; Smirnov, 1981), where the main dynamic variable is the autoionization width $\Gamma_{N}=\tilde{\Gamma}_{N} / R^{6}$ of a pair of interacting atoms. If the atoms are in $N \equiv\left\{N_{d}=n_{d} l_{d}, N_{i}=n_{i} l_{i}\right\}_{\widetilde{\Gamma}}$ quantum states at a fixed internuclear distance $\mathrm{R}$, the reduced Penning width $\tilde{\Gamma}_{N}$ consists of partial widths $\tilde{\Gamma}_{N N^{\prime}}$ corresponding to individual bound-free atom transitions $N \rightarrow N^{\prime}$ (see Fig. 7): $\tilde{\Gamma}_{N}=\sum_{N^{\prime}} \tilde{\Gamma}_{N N^{\prime}}$. The partial widths (a.u.)

$$
\tilde{\Gamma}_{N N^{\prime}}=\left|D_{N_{D}^{\prime} N_{D}}\right|^{2} \frac{c \sigma_{p h}}{\pi\left|\omega_{d}\right|}
$$

include dipole matrix elements $D_{N_{D}^{\prime} N_{D}}$ for the bound-bound d-atom transitions and the photoionization cross section $\sigma_{p h}$ for the i-atom, in accordance with 
its transition into continuum. Frequency of a partial atomic transition $\omega_{d}$ determines the energy that the d-atom transfers to the i-atom (see Fig. 7) in the dipole-dipole interaction. The ionization condition means that $\omega_{d} \geq \varepsilon_{0}$, where the i-atom binding energy $\varepsilon_{0}=1 /\left(2 n_{i}^{* 2}\right)$ is expressed through the effective quantum number $n_{i}^{*}=n_{i}-\delta_{i}$ and the quantum defect $\delta_{i}$. The hatched line in Fig. 7 shows the position of an imaginary level $n_{\text {virt }}$ that is lower than the $n_{d} l_{d}$ state by amount $\varepsilon_{0}$ and, therefore, determines the position of the ionization limit: $n_{d}^{\prime}<n_{\text {virt }}$.

In the Katsuura-Smirnov model, the PI cross-section $\sigma_{P I}$ of two colliding Rydberg atoms, subject to the long-range dipole-dipole interaction, is determined directly by width $\tilde{\Gamma}_{N}$

$$
\sigma_{P I}=3.37 \cdot \tilde{\Gamma}_{N}^{2 / 5} / \mathrm{v}^{2.5},
$$

where $\mathrm{v}$ is the relative velocity of the atoms. Therefore, PI specifics identification is related to the analysis of $\tilde{\Gamma}_{N}$ behavior in various quantum configurations $N$ of atomic pairs.

Publication Bezuglov \& Borodin (1999) suggests simple quasi-classical formulas for calculating radiation constants of optical transitions in alkali-metal atoms, which were used in the surveys of PI autoionization widths $\tilde{\Gamma}_{N}$ for symmetric pairs $\left(n_{d}=n_{i}\right)$ of rubidium atoms (Amthor et al., 2009) and asymmetric pairs $\left(n_{d} \neq n_{i}\right)$ of hydrogen atoms (Efimov et al., 2016). This chapter includes an analysis of PI effects in a cold Rydberg gas of sodium atoms. The accuracy of our presented below numerical results, based on the semi-classical approach, is better than 15\% (Efimov et al., 2016).

\subsection{Optimal Rydberg atomic pairs}

Fig. 8 shows the values of autoionization width $\tilde{\Gamma}_{N}$ as functions of principal quantum numbers $n_{d}, n_{i}$ of $\mathrm{H}$ and $\mathrm{Na}$ atomic pairs. In the first place, a higher PI efficiency for sodium atoms (by an order of magnitude) can be observed. Noteworthy also is a contrasting oscillatory structure $\tilde{\Gamma}_{N}$ present in $n_{d}$ and $n_{i}$ variations. These oscillations belong to PI distinctive features and are caused by the rapid decrease of dipole matrix elements $D_{N_{D}^{\prime} N_{D}}\left(\omega_{d}\right)$ and photoionization cross sections $\sigma_{p h}\left(\varepsilon_{i}^{\prime}\right)$ with the decrease of their arguments (Sobelman, 1992). Therefore, $\tilde{\Gamma}_{N}$ peak corresponds to the occurrence of threshold ionization values $\varepsilon_{i}^{\prime}=0$, which for atomic levels in Fig. 7 corresponds to superimposition of the $\widetilde{n}_{d}^{\prime} l_{d}^{\prime}$ actual state on the $n_{v i r t}$ virtual level. Their divergence leads to the decrease of $\tilde{\Gamma}_{N}$, while the convergence results in its growth, i.e. in oscillations.

Some specific features of surfaces in Fig. 8 include $\tilde{\Gamma}_{N}$ dependence on the quantum number $n_{d}$ at fixed values $n_{i}$ of the ionized i-atom. The set of such dependences is shown for $\mathrm{H}, \mathrm{Na}$ and $\mathrm{K} \mathrm{s}$-s atomic pairs in Fig. 9. A rapid increase of $\tilde{\Gamma}_{N}$ with the de-excited d-atom size $\sim n_{d}^{* 2}$ reduction can be clearly seen.

This is a purely quantum effect caused by an 'average' reduction of $\omega_{d}$ and $\varepsilon_{i}^{\prime}$ arguments for the fuctions $D_{N_{D}^{\prime} N_{D}}, \sigma_{p h}$ with the $n_{d}$ value decrease. For each 


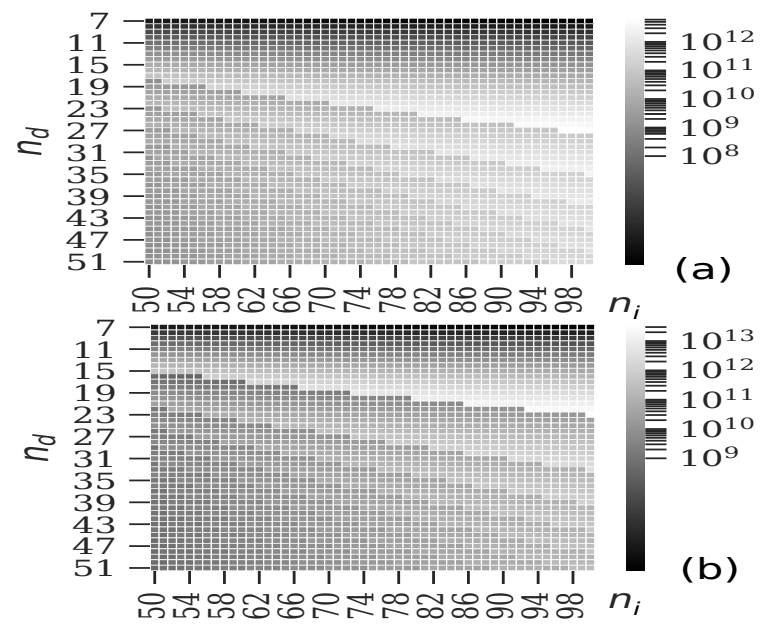

Figure 8. Dependence of reduced autoionization width $\tilde{\Gamma}_{N}$ (at. un.) on principal quantum numbers $n_{d}, n_{i}$ of a diatomic quasi-molecule. The cases of (a) s-s $\left(l_{d}=0, l_{i}=0\right.$ ) configuration for hydrogen atomic pairs and (b) s-p $\left(l_{d}=0, l_{i}=1\right)$ configuration for sodium atoms have been considered.

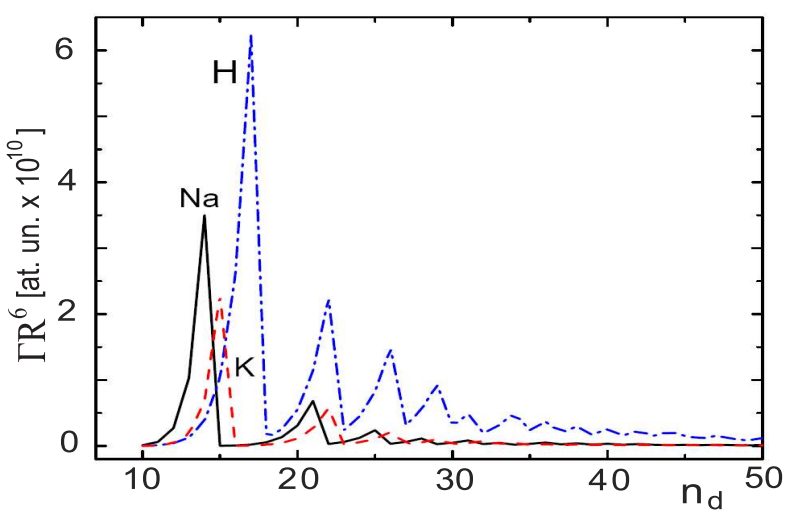

Figure 9. Reduced autoionization widths $\tilde{\Gamma}_{N}=R^{6} \Gamma_{N}$ for the cases of s-s- $\left(l_{d}=0, l_{i}=0\right) \mathrm{H}, \mathrm{Na}$ and $\mathrm{K}$ atomic pairs configurations at the fixed value $n_{i}=50$.

type of atomic pairs presented in Fig. 9 an optimal $n_{d}^{(o p t)}\left(n_{i}\right)$ value for the iatom exists when the maximum peak of $\tilde{\Gamma}^{(o p t)}\left(n_{i}\right)$ occurs. The occurrence of the optimal pair $\left(n_{d}^{(o p t)}, n_{i}\right)$ corresponds, thus, to the coincidence of the energy of virtual level $n_{\text {virt }}$ and the energy $\tilde{\varepsilon}_{d}^{\prime}$ of $\tilde{n}_{d}^{\prime} l_{d}^{\prime}$ state, which is the nearest to 
$n_{d}^{(o p t)} l_{d}$ initial state (see Fig. 7):

$$
\varepsilon_{d}^{(o p t)}-\tilde{\varepsilon}_{d}^{\prime}=\gamma_{d}^{(\min )}\left(n_{d}^{(o p t)}-\delta_{l_{d}}\right)^{-3}=\left(n_{i}-\delta_{l_{i}}\right)^{-2} / 2 .
$$

The positive parameter $\gamma_{d}^{(\min )}>0$ assumes the minimum value for the difference between effective quantum numbers for $l_{d^{-}}$and $l_{d^{\prime}}^{\prime}$-series. Relation (17) describes well the position of $n_{d}^{(o p t)}$ for all the three atomic types shown in Fig. 9.

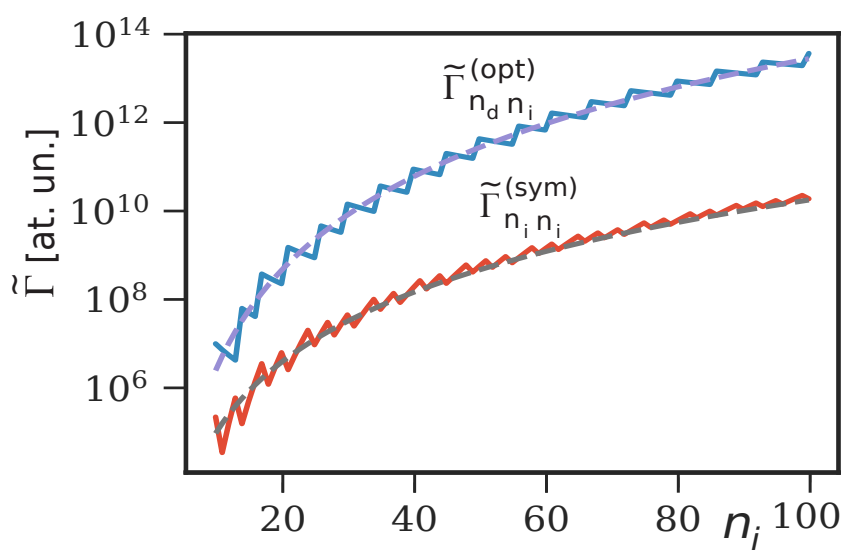

Figure 10. The reduced PI widths $\tilde{\Gamma}_{N}$ for symmetric (sim) and optimal (opt) pairs in relation to the principal quantum number $n_{i}$ of the ionized atom. The case of s-p $\left(l_{d}=0, l_{i}=1\right)$ configuration for sodium atoms pairs has been considered. The dashed curves correspond to the analytical results.

The order of magnitude of PI efficiency increase when a symmetric pair $\left(n_{d}=n_{i}, n_{i}\right)$ is replaced by an optimal pair $\left(n_{d}^{(o p t)}, n_{i}\right)$ is shown in Fig. 10. The respective gain $\Lambda=\tilde{\Gamma}^{(\text {opt })} / \tilde{\Gamma}^{(\text {sym })}$ when $n_{i} \sim 100$ achieves three orders of magnitude.

\subsection{Analytical results}

Quasi-classical representations of partial values $\tilde{\Gamma}_{N N^{\prime}}(15)$ allow obtaining analytical assessment of PI processes efficiency for individual, yet important from the practical standpoint cases of $\Gamma_{N^{\prime}}$ autoionization widths for symmetric/ optimal pairs. Omitting the rather cumbersome calculations, which will be included in another publication Abo et al. (In progress), we shall provide only the final 
results:

$$
\begin{gathered}
\left\langle\widetilde{\Gamma}_{n_{d} n_{i}}^{(s i m)}\right\rangle\left(n_{i}\right) \simeq \Pi_{l_{d} l_{i}}^{(s y m)} \frac{1+34 \sqrt{2} /\left(3 n_{i}^{*}\right)}{\left(1+4 \sqrt{2} \cdot \Xi_{l_{d} l_{i}}^{(s y m)} / n_{i}^{*}\right)^{17 / 3}} n_{i}^{* 16 / 3} \\
\left\langle\widetilde{\Gamma}_{n_{d} n_{i}}^{(o p t)}\right\rangle\left(n_{i}\right) \simeq \Pi_{l_{d} l_{i}}^{(o p t)} \frac{\left(1-\Xi_{l_{d} l_{i}}^{(o p t)} / n_{i}^{* 2 / 3}\right)^{4}}{\left(1+3 \cdot \Xi_{l_{d} l_{i}}^{(o p t)} / n_{i}^{* 2 / 3}\right)^{10 / 3}} n_{i}^{* 19 / 3} .
\end{gathered}
$$

Parameters $\Pi_{l_{d} l_{i}}^{(s i m / o p t)}, \Xi_{\left.l_{d} l_{i} / o p t\right)}^{(s i m c l u d e d ~ i n ~ f o r m u l a s ~(18) ~ a n d ~(19) ~ a r e ~ f u n c-~}$ tions of only orbital $\left\{l_{d}, l_{i}\right\}$ configurations of pairs. The former parameter type (П) regulates the numeric values of PI rate constants. In the case of hydrogen atoms both parameters $\Pi_{l_{d} l_{i}}^{(s i m / o p t)}$ prove to be constant, i.e. $\Pi_{l_{d} l_{i}}^{(s i m)}=1.070$; $\Pi_{l_{d} l_{i}}^{(o p t)}=1.899$, and do not depend on $\left\{l_{d}, l_{i}\right\}$. The latter parameter type $(\Xi)$, which is nondimensional, is responsible for the oscillatory structure (see Fig. 10) of PI rate constants. Concerning oscillation-averaged values $\left\langle\tilde{\Gamma}_{N}\right\rangle$ of Penning widths, the value $\Xi_{l_{d} l_{i}}^{(s i o p t)}=0.5$ could be assumed in the first approximation.

Table 1. $\Pi$ (a.u.) and $\Xi$ parameters values for primary configurations of sodium atomic pairs. For reference: 1 a.u. $=0.906 \cdot 10^{-33} \mathrm{~cm}^{6} / \mathrm{s}$.

\begin{tabular}{lllllllll} 
& s-s & s-p & s-d & s-f & p-s & p-p & p-d & p-f \\
\hline \hline$\Pi^{(s i m)}$ & 0.127 & 0.449 & 0.318 & 0.433 & 0.0992 & 0.34 & 0.244 & 0.332 \\
$\Xi^{(\text {sim })}$ & 0.69 & 0.41 & 0.11 & 0.11 & 1.4 & 0.96 & 0.69 & 0.69 \\
$\Pi^{(o p t)}$ & 2.53 & 9.266 & 6.17 & 8.87 & 0.927 & 3.64 & 2.41 & 3.29 \\
$\Xi^{(o p t)}$ & 0.66 & 0.59 & 0.41 & 0.5 & 0.65 & 0.69 & 0.48 & 0.5 \\
& d-s & d-p & d-d & d-f & f-s & f-p & f-d & f-f \\
\hline \hline$\Pi^{(s i m)}$ & 0.356 & 1.24 & 0.886 & 1.23 & 0.3 & 1.03 & 0.754 & 1.08 \\
$\Xi^{(s i m)}$ & 0.98 & 0.69 & 0.41 & 0.5 & 0.98 & 0.59 & 0.41 & 0.61 \\
$\Pi^{(o p t)}$ & 0.71 & 2.73 & 1.9 & 2.51 & 0.61 & 1.81 & 1.25 & 1.71 \\
$\Xi^{(\text {opt })}$ & 0.5 & 0.43 & 0.3 & 0.31 & 0.82 & 0.41 & 0.32 & 0.31 \\
\hline
\end{tabular}

In the general case of arbitrary atoms of alkali-metal elements, both parameter types $\Pi, \Xi$ can be considered adjustable, while formulas (18) and (19) can be used for rapid estimation of PI autoionization widths. Tab. 1 includes, by way of example, data on $\Pi-, \Xi$-values for the primary orbital configurations of $\mathrm{Na}$ atomic pairs. The 'average' quantity of such calculations can be assessed by the data shown in Fig. 10, where the hatched curves were obtained with the aid of formulas (18) and (19) using the data for s-p pair parameters from Tab. 1. 


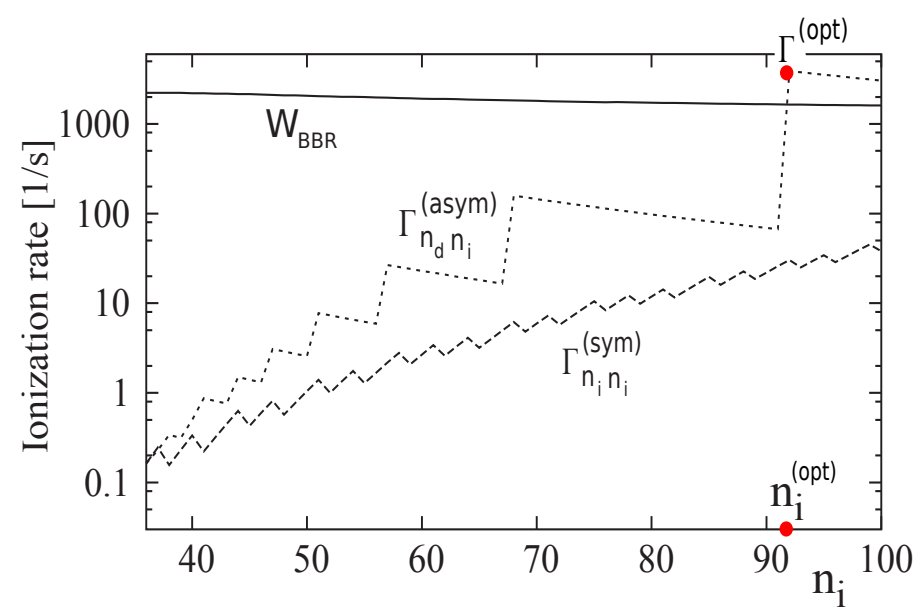

Figure 11. Growth of Penning ionization rates $(\Gamma)$ during diffusion of the atomic population. The black-body radiation-induced ionization rate $\mathrm{W}_{\mathrm{BBR}}$ (Efimov et al., 2016) is exhibited as a solid line as well.

\subsection{Role of asymmetric atomic pairs in cold Rydberg gases}

Both the collisions with electrons and the evolution of population of Rydberg atom pairs via resonant energy transfer lead to the diffusion of the atomic population over a range of Rydberg states (see Fig. 6). Upon this diffusion following the laser excitation of Rydberg hydrogen atomic pairs with $n_{i_{0}}=n_{d_{0}}=n_{0}=36$ and the concentration $\rho_{0}=2.3 \cdot 10^{11} \mathrm{~cm}^{-3}$ (see Fig. 11), the growth of one of the initial quantum numbers $n_{0} \rightarrow n_{i}$ may increase the initial autoionization rate $\Gamma_{n_{0} n_{0}}^{(\operatorname{sim})}$ by three orders of magnitude, while the simultaneous lowering of the other quantum number $n_{0} \rightarrow n_{d}$ can increase $\Gamma_{n_{i} n_{i}}^{(s i m)}$ by another two or three orders of magnitude (see Fig. 11). The aggregated gain of $\Gamma_{n_{d} n_{i}}^{(\text {asim) }}$ compared to the initial rate $\left(\sim 0.1 s^{-1}\right)$ can be as large as $\sim 10^{4}-10^{5}$, and this may strongly enhance the role of PI in the destabilization of cold Rydberg gases via formation of cold plasmas.

The shown findings of the analysis of PI effects in cold Rydberg media allow drawing the following conclusions. Contrary to hydrogen atoms, alkali element atoms feature strong dependence of PI processes efficiency on orbital quantum numbers (see Tab. 1). This is related to the wide variety of $\delta_{l}$ quantum defects of atomic series. Values of parameters $\Pi^{(o p t)}$ in the majority of the cases (see Tab. 1) exceed the "hydrogen reference" value 1.899. Therefore, PI processes involving highly excited states of all atoms of alkali-metal elements may play a dominant role as sources of primary charged particles at the diffusion stage of a cold Rydberg gas evolution into a cold plasma. 


\section{Conclusion}

The paper provides the results of research into the features of autoionization processes in highly excited atomic complexes associated with the evolution of the orbital moment $L$ of Rydberg electrons (RE). Two types of interaction between the complex particles are considered. The first type of interaction is characteristic of reactions of the associative ionization type. It is caused by the charge exchange processes inside the complex ionic core, which results in a quasimonochromatic microwave electric field. The latter perturbs the motion of $\mathrm{RE}$ on the Keplerian orbit with the principal quantum number $n$ and leads to its departure to the energy continuum. We have simulated such type of ionization for a hydrogen atom under the influence of external radiation of frequency $\omega_{0}$ and amplitude $E_{0}$. The results of the analytical consideration in the adiabatic approximation are given. A nontrivial evolution of the orbital moment, having oscillations in both the direction and the absolute value of $L$ with the maximum possible amplitude equal to $n+0.5$, has been discovered. Violation of adiabaticity causes the appearance of irregular motion zones in the RE energy space. When $E_{0}$ exceeds the critical value $E_{c}(12)$, these zones merge into a single whole with a departure to the energy continuum. The evolution of $L$ continues to demonstrate a complex oscillation structure with a slightly suppressed amplitude $\approx 2 n / 3$ of the oscillations. The latter turns out to be less than the critical value $L_{c} \sim n$, and does not lead to any violation of conditions for the one-dimensional diffusion ionization model feasibility.

The second type of interaction between Rydberg particles - dipole-dipole interaction - dominates in ultra-cold gaseous media. The non-threshold transfer of excitation energy between REs leads to the resonance Penning autoionization (PI) of atomic pairs. An important feature of PI is the nontrivial dependence of the rate constants on both the size $\left(\sim n^{2}\right)$ and the orbital moments of the atoms. We have found optimal, highly asymmetric configurations of Rydberg atomic pairs for all alkali metals, leading to explosive intensification (by several orders of magnitude) of free electron formation due to PI processes. This property makes PI an important source of primary charged particle formation when cold Rydberg plasma is created.

Acknowledgements. This work is made within projects of the Ministry of Education, Science and Technological Development of Serbia, grant numbers 176002, III44002

\section{References}

Abo, Z., Miculis, K., Bruvelis, M., et al., Strong enhancement of Penning ionization in cold Rydberg gases II: Tom and Jerry pairs for alkali-metal atoms. In progress, J. Phys. B 
Amthor, T., Denskat, J., Giese, C., et al., Autoionization of an ultracold Rydberg gas through resonant dipole coupling. 2009, Eur. Phys. J. D, 53, 329

Bezuglov, N. \& Borodin, V., Generalized correspondence rules for quasi-classical dipole matrix elements. 1999, Opt. Spectrosc., 86, 467

Bezuglov, N., Borodin, V., Eckers, A., \& Klyucharev, A., A quasi-classical description of the stochastic dynamics of a Rydberg electron in a diatomic quasi-molecular complex. 2002, Opt. Spectrosc., 93, 661

Bezuglov, N., Borodin, V., Kazanskii, A., et al., Analysis of Fokker-Planck type stochastic equations with variable boundary conditions in an elementary process of collisional ionization. 2001, Opt. Spectrosc., 91, 19

Buenker, R. J., Golubkov, G., Golubkov, M., Karpov, I., \& Manzheliy, M., Relativity laws for the variation of rates of clocks moving in free space and GPS positioning errors caused by space-weather events. 2013, in Global Navigation Satellite SystemsFrom Stellar to Satellite Navigation (IntechOpen)

Chu, S.-I. \& Telnov, D. A., Beyond the Floquet theorem: generalized Floquet formalisms and quasienergy methods for atomic and molecular multiphoton processes in intense laser fields. 2004, Phys. Rep., 390, 1

Dalgarno, A. 1975, in book Rydberg States of Atoms and Molecules, by R. F. Stebbings , F. B. Dunning, Cambridge, UK: Cambridge University Press

Dashevskaya, E., Litvin, I., Nikitin, E., Oref, I., \& Troe, J., Classical diffusion model of vibrational predissociation of van der Waals complexes Part III. Comparison with quantum calculations. 2002, Phys. Chem. Chem. Phys., 4, 3330

Delone, N. B., Krainov, B., \& Shepelyanskii, D., Highly-excited atoms in the electromagnetic field. 1983, Sov. Phys. Usp., 26, 551

Efimov, D., Bezuglov, N., Klyucharev, A., et al., Analysis of light-induced diffusion ionization of a three-dimensional hydrogen atom based on the Floquet technique and split-operator method. 2014, Opt. Spectrosc., 117, 8

Efimov, D., Miculis, K., Bezuglov, N., \& Ekers, A., Strong enhancement of Penning ionization for asymmetric atom pairs in cold Rydberg gases: the Tom and Jerry effect. 2016, J. Phys. B, 49, 125302

Fermi, E., Sopra lo spostamento per pressione delle righe elevate delle serie spettrali. 1934, Il Nuovo Cimento (1924-1942), 11, 157

Gnedin, Y. N., Mihajlov, A. A., Ignjatović, L. M., et al., Rydberg atoms in astrophysics. 2009, New Astron. Rev., 53, 259

Golubkov, G. \& Devdariani, A., Associative ionization in slow collisions of atoms. 2011, Russ. J. Phys. Chem. B, 5, 892

Graham, W. G., Fritsch, W., Hahn, Y., \& Tanis, J. Recombination of atomic ions, , Vol. 296 (Springer Science \& Business Media)

Hairer, E., Numeral geometric integration. 1999, Universite de Geneve, Geneve

Janev, R. \& Mihajlov, A., Resonant ionization in slow-atom-Rydberg-atom collisions. 1980, Phys. Rev. A, 21, 819 
Katsuura, K., Remarks on excitation transfer between unlike molecules. 1965, J. Chem. Phys., 43, 4149

Kazansky, A., Bezuglov, N., Molisch, A., Fuso, F., \& Allegrini, M., Direct numerical method to solve radiation trapping problems with a Doppler-broadening mechanism for partial frequency redistribution. 2001, Phys. Rev. A., 64, 022719

Kirova, T., Cinins, A., Efimov, D., et al., Hyperfine interaction in the Autler-Townes effect: The formation of bright, dark, and chameleon states. 2017, Phys. Rev. A, 96, 043421

Klyucharev, A., Bezuglov, N., Matveev, A., et al., Rate coefficients for the chemiionization processes in sodium-and other alkali-metal geocosmical plasmas. 2007, New Astron. Rev., 51, 547

Koch, P. \& Van Leeuwen, K., The importance of resonances in microwave ionization of excited hydrogen atoms. 1995, Phys. Rep, 255, 289

Krainov, V., Ionization of atoms in strong low-frequency electromagnetic field. 2010, J. Exp. Theor. Phys., 111, 171

Landau, L. \& Lifshitz, E., Mechanics (Nauka, Moscow, 1973); English tran., Course of theoretical physics. Vol. 1. Mechanics. 1976, Permagon Press, Oxford-New YorkToronto

Landau, L. \& Lifshitz, E. 1977, Quantum Mechanics Pergamon Press

Lopes, R. M. \& Williams, D. A., Io after Galileo. 2005, Rep. Prog. Phys., 68, 303

Lyon, M. \& Rolston, S., Ultracold neutral plasmas. 2016, Rep. Prog. Phys., 80, 017001

Mihajlov, A. A., Ignjatović, L. M., Dimitrijević, M. S., \& Djurić, Z., Symmetrical Chemi-Ionization and Chemi-Recombination Processes in Low-Temperature Layers of Helium-rich DB White Dwarf Atmospheres. 2003, Astrophys. J. Suppl., 147, 369

Saffman, M., Walker, T. G., \& Mølmer, K., Quantum information with Rydberg atoms. 2010, Rev. Mod. Phys., 82, 2313

Smirnov, B. M., lonization in low-energy atomic collisions. 1981, Soviet Physics Uspekhi, 24, 251

Sobelman, I. Atomic spectra and radiative transitions, , Vol. 12 (Springer Science \& Business Media)

Sydoryk, I., Bezuglov, N., Beterov, I., et al., Broadening and intensity redistribution in the $\mathrm{Na}(3 \mathrm{p})$ hyperfine excitation spectra due to optical pumping in the weak excitation limit. 2008, Phys. Rev. A., 77, 042511

Tanner, P. J., Han, J., Shuman, E., \& Gallagher, T., Many-body ionization in a frozen Rydberg gas. 2008, Phys. Rev. Lett., 100, 043002

Zaslavsky, G. M. 2007, The Physics of Chaos in Hamiltonian Systems (the 2dn Edition Imperial College Press) 